\title{
BMJ Open Impact of general anaesthesia in overall and disease-free survival compared to other types of anaesthesia in patients undergoing surgery for cutaneous melanoma: a systematic review and meta-analysis protocol
}

\author{
Bruno Luís de Castro Araujo, ${ }^{1}$ Jadivan Leite de Oliveira, ${ }^{2}$ \\ Flavia de Miranda Corrêa, ${ }^{3}$ Luis Eduardo Santos Fontes, ${ }^{4}$ \\ Andreia Cristina de Melo, ${ }^{5}$ Luiz Claudio Santos Thuler ${ }^{5,6}$
}

To cite: Araujo BLC, de Oliveira JL, Corrêa FM, et al. Impact of general anaesthesia in overall and disease-free survival compared to other types of anaesthesia in patients undergoing surgery for cutaneous melanoma: a systematic review and metaanalysis protocol. BMJ Open 2019;9:e027993. doi:10.1136/ bmjopen-2018-027993

- Prepublication history and additional material for this paper are available online. To view these files, please visit the journal online (http://dx.doi. org/10.1136/bmjopen-2018027993).

Received 17 November 2018 Revised 22 May 2019 Accepted 3 July 2019

Check for updates

(C) Author(s) (or their employer(s)) 2019. Re-use permitted under CC BY-NC. No commercial re-use. See rights and permissions. Published by BMJ.

For numbered affiliations see end of article.

Correspondence to Bruno Luís de Castro Araujo; brunoaraujomed@yahoo.com.br

\begin{abstract}
Introduction Cutaneous melanoma is an aggressive type of skin cancer. Anaesthetic agents may have an impact on the immune response, postoperative neurohumoral response and tumour progression. This systematic review aims to evaluate the impact of general anaesthesia on overall and disease-free survival compared with other types anaesthesia in patients undergoing surgery for cutaneous melanoma.
\end{abstract}

Methods and analysis The review will analyse data from controlled and observational studies of patients undergoing surgery for melanoma under general anaesthesia compared with other types of anaesthesia. The primary outcomes are overall survival and disease-free survival. The secondary outcomes are health-related quality of life, time to tumour progression, distant disease-free survival, time to treatment failure, cancer-specific survival, biochemical recurrence, return of intended oncological therapy, days alive and out of the hospital at 90 days, cost analysis and adverse events. A comprehensive literature search will be performed using the MEDLINE, EMBASE, Cochrane CENTRAL, Web of Science, LILACS and IBECS databases. Grey literature will also be searched. Risk of methodological bias will be assessed using The Cochrane Collaboration's revised tool for assessing risk of bias in randomised trials (RoB 2.0) and the NewcastleOttawa scale. Two reviewers will independently assess the eligibility of studies and risk of bias; a third author will solve discrepancies. One author will perform data extraction and the other will check the process and data. Qualitative analysis will be carried out using all included studies. A meta-analysis using a random-effects model for pooled risk estimates will be carried out for the two main outcomes and for selected secondary outcomes if they conform to previously stated criteria. The GRADE approach will be used to summarise the quality of evidence.

Ethics and dissemination Ethics approval is not required as we analyse data from previously reported studies. PROSPERO registration number CRD42018114918.
Strengths and limitations of this study

- This will be the first comprehensive systematic review designed specifically to assess the impact of anaesthetic technique on overall and disease-free survival in melanoma.

- The inclusion of non-randomised studies is both a strength and a limitation of the protocol.

- Observational studies will not be combined with randomised controlled trials and quasi-randomised trials, limiting the influence of study design on the effects measured in this meta-analysis.

- A rigorous and sensitive search will be performed to maximise comprehensiveness and minimise bias.

- The Grading of Recommendations, Assessment, Development and Evaluation approach will be used to inform conclusions in an appropriate manner.

\section{INTRODUCTION}

Cutaneous melanoma is the most lethal form of skin cancer. ${ }^{1}$ It is the 21 st most frequent cancer worldwide with a rising incidence, probably due to the increase in life expectancy. ${ }^{2}$ Early stages of melanoma may be cured by excision of primary lesion, but advanced disease is still a challenge despite the recent advances in treatment. There are many factors that lead to a recurrence of cutaneous melanoma after primary surgery. The main prognostic factors are the histological type, Breslow depth, cutaneous layer invasion (Clark level), regression, mitosis, ulceration on primary lesion, satellite and 'in transit' lesions, lymphatic involvement and metastatic spread. ${ }^{3}$

Recently, the impact of the anaesthetic technique on recurrence rates of many types 
of tumours has been a point of intense debate. Retrospective clinical evidence has found a protective effect of some anaesthetics over others in many tumour types, including, but not limited to colon, ${ }^{4}$ breast, ${ }^{5}$ laryngeal, ${ }^{6}$ ovarian, ${ }^{7}$ prostate, ${ }^{8}$ bladder $^{9}$ and cutaneous melanoma. ${ }^{10}$

Surgery can activate the sympathetic nervous system and the hypothalamic-pituitary-adrenal axis. ${ }^{11}$ This leads to an increase in the sympathetic tone, release of adrenocorticotropic hormone and synthesis of corticosteroids and catecholamines by the adrenal gland. ${ }^{11}$ Thus, surgery is considered to be an important contributory factor for the clinical evolution of cancer. Inhalational anaesthetics are being investigated as an important facilitator for perioperative tumour dissemination. ${ }^{12}$ They may cause inhibition of cellular immunity and promote angiogenesis and cellular proliferation. ${ }^{13}$ Basic research in anaesthetic-induced organ protection provides important information regarding cellular signalling, especially, hypoxia-inducible factors (HIFs).$^{14}$ Halogenated inhalational anaesthetics can induce HIFs, possibly resulting in a cardiac, cerebral, hepatic and renal cytoprotection described as 'anaesthetic preconditioning'. ${ }^{14}$ The HIF system is essential for adaptation to the reduced supply of oxygen to healthy cells; however, it also helps the continued survival of tumour cells. ${ }^{14}$ There is a large body of evidence regarding the relationship of HIFs with cancer. ${ }^{15}$

Experimental data support the hypothesis of anaesthetics influencing melanoma cells. Exposure to halothane and isoflurane, when compared with oxygen, was correlated to an increased number of lung metastasis in C57BL mice model injected with B16 melanoma cells. ${ }^{16}$ In contrast, propofol induced apoptosis of B16F10 melanoma cells 'in vitro'. ${ }^{17}$ Lidocaine and ropivacaine reduced the viability of melanoma cells and increased apoptosis in a concentration-dependent manner 'in vitro'. ${ }^{8}$ The first report of impaired survival associated with the use of general anaesthesia for melanoma surgery was published by Seebacher et al; subsequent investigators achieved conflicting results..$^{10-21}$

Changes in institutional anaesthesia protocols to avoid general anaesthesia can impact the cost and the overall safety of surgical procedure. Therefore, a systematic review and analysis of overall and disease-free survival may modify clinical practice. This systematic review may influence the choice of anaesthetic technique among anaesthetists, dermatologists, surgical oncologists and patients.

The main objective of the proposed study is to evaluate the relationship between the anaesthetic technique and the overall and disease-free survival of malignant melanoma patients undergoing surgical resection. The question formulated to fulfil the study objective is: does general anaesthesia imply worse overall or disease-free survival rate compared with other types of anaesthesia in patients undergoing surgery for cutaneous melanoma? The secondary objectives are assessment of health-related quality of life, time to tumour progression, distant disease-free survival, time to treatment failure, cancer-specific survival, biochemical recurrence, return of intended oncological therapy, days alive and out of the hospital at 90 days, costs and adverse events.

This systematic review protocol was designed in accordance with the Preferred Reporting Items for Systematic Reviews and Meta-Analyses Protocols (PRISMA-P) statement. ${ }^{22}$ The Meta-analysis of Observational Studies in Epidemiology (MOOSE) proposal for reporting observational studies was also used as a reference for protocol development. ${ }^{23}$ This systematic review has no specific funding. The systematic review protocol was registered with the International Prospective Register of Systematic Reviews (PROSPERO) on 16 November 2018 and has not been updated (registration number CRD42018114918). A protocol amendment with the modifications of the systematic review protocol following the peer review during the BMJ Open editorial process will be described in detail, including the date and the rationale; this will be reported in the PROSPERO database.

\section{METHODS AND ANALYSIS \\ Eligibility criteria \\ Participants}

The systematic review will include human studies evaluating patients undergoing surgery for cutaneous melanoma. Non-cutaneous melanomas will not be included in the review. If the term 'melanoma' is included in the text of the manuscript, it will be assumed to imply cutaneous melanoma, since it is the most frequent subtype of the disease. Studies with fewer than 10 participants on each arm will be excluded. No age, sex, or race restrictions will be applied. In case of studies that involve the overlap of patients, only the most recent article will be chosen for inclusion.

\section{Study design}

Randomised controlled trials (RCTs), quasi-randomised trials and non-randomised studies (cohort and casecontrol studies) will be included in the final analysis.

\section{Interventions}

To be included in the review, the study must report a comparison of patients who underwent general anaesthesia with other types of anaesthesia. Techniques other than general anaesthesia will be aggregated as a single group in each study.

\section{Outcomes}

The aim is to assess if the use of general anaesthesia results in a higher risk of death or recurrence in melanoma patients. The primary outcomes are overall survival and disease-free survival. The secondary ouctomes are health-related quality of life, time to tumour progression, distant disease-free survival, time to treatment failure, cancer-specific survival, biochemical recurrence, return of intended oncological therapy, days alive and out of the hospital at 90 days, cost analysis and adverse events. Outcomes are not part of the eligibility criteria to be 
included in the review. Results of individual studies not including predefined outcomes will be reported in the body of the article or in an appendix according to the authors conclusions regarding the relevance of individual studies.

\section{Timing}

No timing restriction will be applied. All potentially relevant articles available in the selected databases will be included in the review.

\section{Setting and language}

The initial triage of articles will require a title in English. No other language restrictions will be applied and articles in other languages will be translated when necessary for analysing eligibility criteria, evaluating risk of bias and data extraction. The authors of the original articles will be contacted when deemed necessary, first by email, and then through other digital platforms (eg, LinkedIn, ORCID and ResearchGate) and correspondence.

\section{Information sources}

The main electronic databases accessed will be MEDLINE (PubMed interface), Excerpta Medica database (EMBASE), Cochrane Central Register of Controlled Trials (CENTRAL), Web of Science (online search engine, using all available databases), Latin American and Caribbean Health Sciences Literature (in Portuguese: Literatura Latino-Americana e do Caribe em Ciências da Saúde-LILACS) and The Spanish Bibliographic Index of the Health Sciences (in Spanish: Índice Bibliográfico Español en Ciencias de la Salud-IBECS). We will include studies published from the start of indexing until 30 October 2018.

\section{Other sources}

Hand searches of the first 200 citations on Google Scholar will be performed. Reference lists of the included articles, reviews and citing articles searched using the Web of Science database will be checked. Grey literature will be searched using the Open Grey (http://www.opengrey.eu) and the Open Access Theses and Dissertations (https://oatd.org) registries. The International Clinical Trials Registry Platform search portal (http://apps.who. int/trialsearch) will also be accessed.

\section{Search strategy}

Search terms are designed to address the Patient, Intervention, Comparison, Outcome standards. Patients will be searched using melanoma-related terms. For interventions and comparisons, anaesthesia related terms will be used. The authors of the systematic review decided to exclude the outcomes and any specific term related to the study design to increase the sensitivity of the search strategy. The specific search strategies were developed by one author (BLCA) and reviewed by a Health Science Librarian with expertise in systematic review searches. MEDLINE, EMBASE and LILACS searches were chosen according to specific Medical Subject Headings (MeSH),

\begin{tabular}{ll}
\hline Table 1 & PubMed search strategy \\
\hline Database & Search \\
\hline PubMed & 1. Anesthesia[MeSH Terms] \\
& 2. Anesthetics[MeSH Terms] \\
& 3. Anesthesiology[MeSH Terms] \\
& 4. Anest ${ }^{*}$ Title/Abstract] \\
& 5. Anaest* ${ }^{*}$ Title/Abstract] \\
& 6. Analg*[Title/Abstract] \\
& 7. \#1 OR \#2 OR \#3 OR \#4 OR \#5 OR \#6 \\
& 8. Melanoma[MeSH Terms] \\
9. Melanoma*[Title/Abstract] \\
10. \#8 OR \#9 \\
11. \#7 AND \#10
\end{tabular}

Embase subject headings (Emtree) and Health Sciences Descriptors (in Portuguese: Descritores em Ciências da Saúde-DeCS) terms, respectively. The search strategy for PubMed is described in table 1 and the complete search strategies are reported in Appendix 1.

\section{Data management}

EndNote web will be used for reference management; Rayyan (Qatar Computing Research Institute) web application will be used for the process of selection of studies. Cochrane Collaboration's Review Manager (RevMan) software and R software will be used for systematic review data management and statistical analysis.

\section{Selection of studies}

Two authors (BLCA and JLO) will check all the references in the databases. Independent evaluation will be carried out using a stepwise approach for screening, eligibility and inclusion of studies. Inter-rater agreement within the screening process will be assessed by using Cohen's kappa statistic in each step and reported. ${ }^{24}$ In the screening phase, articles selected by at least one of the authors will be submitted to full-text evaluation in the eligibility phase if a consensus is not reached between authors. Disagreements will be resolved by consensus or at the discretion of the senior researcher (LCST). One review author (BLCA) will extract the data to the RevMan software and a second author (JLO) will check the process and the data collected.

\section{Risk of bias}

The Cochrane Collaboration's revised tool for assessing the risk of bias in randomised trials (RoB 2.0) will be used to evaluate RCTs; the Newcastle-Ottawa scale will be used to assess methodological bias in observational studies. The risk of bias assessment will be conducted by two authors (BLCA and JLO); in case of disagreement, a third author (LCST) will arbitrate. The summary of the assessment of the risk of bias in each category will be reported.

\section{Publication bias}

If 10 or more studies are included in the systematic review, a funnel plot visual analysis will be performed for publication bias assessment. 


\section{Heterogeneity}

Statistical heterogeneity will be assessed using $\left(\chi^{2}\right)$ and inconsistency $\left(\mathrm{I}^{2}\right)$ tests. Heterogeneity will be quantified by the $\mathrm{I}^{2}$ test described in the Cochrane Handbook for Systematic Reviews of Interventions and will be reported as low $\left(\mathrm{I}^{2}=0 \%-25 \%\right)$, moderate $\left(\mathrm{I}^{2}=26 \%-\right.$ $50 \%)$, or high $\left(\mathrm{I}^{2}>50\right) .{ }^{24}$ If, according to the judgement of the reviewers, clinical, methodological and statistical heterogeneities make pooling of data inappropriate for a specific outcome, the meta-analysis will be omitted for this outcome. However, data of individual studies will be displayed as a forest plot for a better appraisal of the results.

\section{Qualitative analysis}

The studies included in the review evaluating the primary and secondary outcomes will be summarised in tables including authorship, year of publication, study sample, design, interventions or arms, comparisons, reported outcomes and results. Other details regarding study design and quality of reports will also be described, addressing the strengths and weaknesses of the body of evidence and how they impact the interpretation of the results of the meta-analysis.

\section{Quantitative analysis}

RCTs and quasi-randomised trials will be pooled separately from observational studies for meta-analysis to reduce methodological heterogeneity. Overall and disease-free survival analysis will be quantitatively evaluated if more than one study with the same design is included for a specific endpoint. A meta-analysis will also be performed if more than one study reports the secondary outcomes time to tumour progression, distant disease-free survival, time to treatment failure, cancer specific survival, return of intended oncological therapy and days alive and out of hospital at 90 days. HR estimation will be used as the summary measure for RCTs, quasi-randomised trials and cohort studies; however, days alive out and of the hospital at 90 days will be evaluated using ORs, independent of study design. Case-control studies will be reported using ORs as the summary measure, and the data from this type of study will be reported separately. Effect size will be measured with $95 \%$ CIs, and significance will be set at $\mathrm{p}<0.05$, with the study as the unit of analysis. Adjusted data will be used if available, to reduce the risk of confounding in observational studies. The use of an adjusted estimate has a higher priority than requiring a similar period of follow-up across studies, because reduction of confounding factors is critical in ensuring the generality of the results. If it is not possible to extract HR data from other sources, Kaplan-Meier curves will be the source of the data, using a pixel-coordinate method of mapping the axes of interest and calculation of percentages. If 5 -year overall survival is reported, it will be the preferred follow-up period for HR analysis. When 5-year survival is not reported, we will attempt to contact the authors for this information; if no contact is possible, the longest reported follow-up period will be chosen. Two-year disease-free survival will be used as the other study outcome. These preferred periods of follow-up were chosen in accordance with recent recommendations for analyses of postoperative cancer outcomes. ${ }^{25}$ For the secondary outcomes of timeto-event data, the longest reported follow-up period will be used. Minimum follow-up required to be included in the meta-analysis for the time-to event data is estimated at 2 years. Results will be aggregated independent of the duration of follow-up if longer than 2 years. The inclusion of trials outside the target follow-up period will increase the power of the review without impacting the goals of the review. A broad definition regarding patient selection in studies will be used, permitting the inclusion of different stages, surgical procedures and control groups between studies. A random effects model will be used to perform the meta-analysis, considering the anticipated clinical and methodological heterogeneity. A sensitivity analysis will be performed excluding studies with follow-up periods other than 5 year overall and 2 year disease-free survival. Sensitivity analysis will also be carried out after excluding studies that are judged to have a risk of bias to evaluate the impact of clinical and methodological heterogeneity on outcomes. The year of publication (to assess changes in therapy over time) and the anaesthetic technique used in the control group (local, regional, and both) will be used as parameters to perform a meta-regression and subgroup analysis.

\section{Quality of the body of evidence}

The Grading of Recommendations Assessment, Development and Evaluation (GRADE) approach to summarise the quality of evidence for each outcome will be applied. ${ }^{26}$ The GRADE rating scale assigns high, moderate, low, or very low reliability categories to a body of evidence as detailed elsewhere. ${ }^{26}$

\section{DISCUSSION}

Some of the previous systematic reviews investigating the relationship between exposure to anaesthetic agents on survival and oncological outcomes included different cancer types and anaesthetic agents in the same evaluation. ${ }^{27}$ Cancer cannot be treated as a single disease or a group of diseases with a similar response to various treatment modalities. Therefore, systematic reviews on this topic should consider relevance to specific types of cancer regarding tumour biology and specific surgical techniques employed, despite the lack of prospective studies in this field.

The inclusion of cohort and case-control studies in the systematic review may be an expected source of bias. The association between anaesthetic technique and oncological outcomes is not an anticipated endpoint of therapy; we aim to assess the possibility of unexpected harm in this systematic review. Unequivocal evidence of association of the anaesthetic technique with survival outcomes through RCTs may take several decades to establish. Such studies 
are expensive, take a long period of time and require extensive follow-up. Hence, they are usually outside the scope of regular anaesthesia research. Decision-making is complex in the absence of such high-quality evidence, because evidence of harm is difficult to establish, though harm may occur in some instances. Therefore, observational data must be carefully assessed, especially when prospective data is inadequate. Adjusted data from observational studies by pooled analysis will be used to overcome confounding factors. Observational studies will not be combined with RCTs or quasi-randomised trials, limiting the influence of study design on the effects measured by this meta-analysis.

A recent consensus of experts in the field of anaesthesiology defined the main outcomes to be chosen when evaluating the impact of anaesthesia techniques on cancer outcomes. ${ }^{25}$ The endpoints chosen for this systematic review are based on this report. A uniform definition of outcomes of interest is essential to carry out future observational studies and clinical trial protocols.

\section{ETHICS AND DISSEMINATION}

This study is a systematic review with meta-analysis that evaluates data from previously reported studies; hence ethical approval is not required. We plan to publish this study in a peer-reviewed journal.

\section{Author affiliations \\ ${ }^{1}$ Department of Anaesthesiology, Hospital do Câncer II, National Cancer Institute of Brazil (INCA), Rio de Janeiro, Brazil \\ ${ }^{2}$ Connective and Bone Tissue Section, Hospital do Câncer II, National Cancer Institute of Brazil (INCA), Rio de Janeiro, Brazil \\ ${ }^{3}$ Health Technology Assessment Unit, Population Research Division, National Cancer Institute of Brazil (INCA), Rio de Janeiro, Brazil \\ ${ }^{4}$ Department of Evidence-Based Medicine, Intensive Care, Gastroenterology, Petrópolis Medical School, Rio de Janeiro, Brazil \\ ${ }^{5}$ Clinical Research Division, National Cancer Institute of Brazil (INCA), Rio de Janeiro, Brazil \\ ${ }^{6}$ Postgraduate Program in Neurosciences, Federal University of Rio de Janeiro State, Rio de Janeiro, Brazil}

Acknowledgements We are grateful to acknowledge to Raphael Chanca, the Health Science Librarian who reviewed the systematic review search strategy.

Contributors BLCA is the guarantor of the review and drafted the manuscript. All authors contributed to the inclusion criteria, the risk of bias assessment and data extraction strategies. FMC and LESF contributed with their knowledge on systematic reviews. LCST and FMC will contribute with epidemiological and statistical analysis. ACM contributed through expertise in medical, and JLO, through expertise in surgical oncology. BA contributed with anaesthetic knowledge. BLCA and JLO will screen potential studies, perform duplicate independent data extraction, risk of bias assessment, GRADE assessment. LCST will act as a third reviewer and arbitrator if necessary. All authors read, provided comments and approved the final version of the protocol.

Funding The authors have not declared a specific grant for this research from any funding agency in the public, commercial or not-for-profit sectors.

Competing interests None declared.

Patient consent for publication Not required.

Provenance and peer review Not commissioned; externally peer reviewed.

Open access This is an open access article distributed in accordance with the Creative Commons Attribution Non Commercial (CC BY-NC 4.0) license, which permits others to distribute, remix, adapt, build upon this work non-commercially, and license their derivative works on different terms, provided the original work is properly cited, appropriate credit is given, any changes made indicated, and the use is non-commercial. See: http://creativecommons.org/licenses/by-nc/4.0/.

\section{REFERENCES}

1. Dimitriou F, Krattinger R, Ramelyte E, et al. The world of melanoma: epidemiologic, genetic, and anatomic differences of melanoma across the globe. Curr Oncol Rep 2018;20:87.

2. Bray F, Ferlay J, Soerjomataram I, et al. Global cancer statistics 2018: GLOBOCAN estimates of incidence and mortality worldwide for 36 cancers in 185 countries. CA Cancer J Clin 2018;68:394-424.

3. Gershenwald JE, Scolyer RA, Hess KR, et al. Melanoma staging: evidence-based changes in the American Joint Committee on Cancer eighth edition cancer staging manual. CA Cancer J Clin 2017;67:472-92.

4. Wu ZF, Lee MS, Wong CS, et al. Propofol-based total intravenous anesthesia is associated with better survival than desflurane anesthesia in colon cancer surgery. Anesthesiology 2018;129:932-41.

5. Li R, Liu H, Dilger JP, et al. Effect of propofol on breast cancer cell, the immune system, and patient outcome. BMC Anesthesiol 2018;18:77.

6. Merquiol F, Montelimard AS, Nourissat A, et al. Cervical epidural anesthesia is associated with increased cancer-free survival in laryngeal and hypopharyngeal cancer surgery: a retrospective propensity-matched analysis. Reg Anesth Pain Med 2013;38:398-402.

7. Elias KM, Kang S, Liu X, et al. Anesthetic selection and disease-free survival following optimal primary cytoreductive surgery for stage III epithelial ovarian cancer. Ann Surg Oncol 2015;22:1341-8.

8. Jang D, Lim CS, Shin YS, et al. A comparison of regional and genera anesthesia effects on 5 year survival and cancer recurrence after transurethral resection of the bladder tumor: a retrospective analysis. BMC Anesthesiol 2016;16:16:16.

9. Pei L, Tan G, Wang L, et al. Comparison of combined generalepidural anesthesia with general anesthesia effects on survival and cancer recurrence: a meta-analysis of retrospective and prospective studies. PLoS One 2014;9:e114667.

10. Gottschalk A, Brodner G, Van Aken HK, et al. Can regional anaesthesia for lymph-node dissection improve the prognosis in malignant melanoma? Br J Anaesth 2012;109:253-9.

11. Prete $\mathrm{A}$, Yan $\mathrm{Q}, \mathrm{Al}$-Tarrah $\mathrm{K}$, et al. The cortisol stress response induced by surgery: a systematic review and meta-analysis. Clin Endocrinol 2018;89:554-67.

12. Hooijmans CR, Geessink FJ, Ritskes-Hoitinga M, et al. A systematic review of the modifying effect of anaesthetic drugs on metastasis in animal models for cancer. PLoS One 2016;11:e0156152.

13. Ash SA, Buggy DJ. Does regional anaesthesia and analgesia or opioid analgesia influence recurrence after primary cancer surgery? An update of available evidence. Best Pract Res Clin Anaesthesiol 2013;27:441-56.

14. Tavare AN, Perry NJ, Benzonana LL, et al. Cancer recurrence after surgery: direct and indirect effects of anesthetic agents. Int $J$ Cancer 2012;130:1237-50

15. Soni S, Padwad YS. HIF-1 in cancer therapy: two decade long story of a transcription factor. Acta Oncol 2017;56:503-15.

16. Moudgil GC, Singal DP. Halothane and isoflurane enhance melanoma tumour metastasis in mice. Can J Anaesth 1997;44:90-4.

17. Shang Z, Feng H, Cui L, et al. Propofol promotes apoptosis and suppresses the HOTAIR-mediated mTOR/p70S6K signaling pathway in melanoma cells. Oncol Lett 2018;15:630-4.

18. Kang DK, Zhao LY, Wang HL. Cytotoxic effects of local anesthesia through lidocaine/ropivacaine on human melanoma cell lines. Braz $J$ Anesthesiol 2016;66:594-602

19. Seebacher C, Heubaum F, Küster P, et al. [Comparative analysis of narcosis and local anesthesia in surgery of malignant melanoma of the skin]. Hautarzt 1990;41:137-41.

20. Schlagenhauff $B$, Ellwanger $U$, Breuninger $H$, et al. Prognostic impact of the type of anaesthesia used during the excision of primary cutaneous melanoma. Melanoma Res 2000;10:165-9.

21. Kofler L, Breuninger $\mathrm{H}$, Häfner HM, et al. Lymph node dissection for melanoma using tumescence local anaesthesia: an observational study. Eur J Dermatol 2018;28:177-85.

22. Moher D, Shamseer L, Clarke M, et al. Preferred reporting items for systematic review and meta-analysis protocols (PRISMA-P) 2015 statement. Syst Rev 2015;4:1.

23. Stroup DF, Berlin JA, Morton SC, et al. Meta-analysis of observational studies in epidemiology: a proposal for reporting. Meta-analysis Of Observational Studies in Epidemiology (MOOSE) group. JAMA 2000;283:2008-12. 
24. Higgins JPT, Green S, eds. Cochrane handbook for systematic reviews of interventions version 5.1.0: The Cochrane Collaboration, 2011.

25. Buggy DJ, Freeman J, Johnson MZ, et al. Systematic review and consensus definitions for standardised endpoints in perioperative medicine: postoperative cancer outcomes. Br J Anaesth 2018;121:38-44.

26. Guyatt GH, Oxman AD, Kunz R, et al. What is "quality of evidence" and why is it important to clinicians? BMJ 2008;336:995-8.
27. Soltanizadeh S, Degett TH, Gögenur I. Outcomes of cancer surgery after inhalational and intravenous anesthesia: a systematic review. J Clin Anesth 2017;42:19-25.

28. Sun Y, Li T, Gan TJ. The effects of perioperative regional anesthesia and analgesia on cancer recurrence and survival after oncology surgery: a systematic review and meta-analysis. Reg Anesth Pain Med 2015;40:589-98. 